APS/123-QED

\title{
Angle-dependent $\mathrm{Ni}^{2+}$ x-ray magnetic linear dichroism: Interfacial coupling revisited
}

Elke Arenholz, ${ }^{1, *}$ Gerrit van der Laan, ${ }^{2, \dagger}$ Rajesh V. Chopdekar, ${ }^{3, \ddagger}$ and Yuri Suzuki ${ }^{3}$

${ }^{1}$ Advanced Light Source, Lawrence Berkeley National Laboratory, Berkeley, CA 94720

2Magnetic Spectroscopy Group, Daresbury Laboratory, Warrington WA4 4AD, United Kingdom

${ }^{3}$ Department of Materials Science and Engineering, UC Berkeley, Berkeley, CA 94720

(Dated: May 11, 2007)

\begin{abstract}
Using x ray magnetic linear dichroism (XMLD) for magnetometry requires detailed knowledge of its dependence on the relative orientation of polarization, magnetic moments, and crystallographic axes. We show that $\mathrm{Ni}^{2+} L_{2,3}$ XMLD in cubic lattices has to be described as linear combination of two fundamental spectra - not one as previously assumed. The spectra are calculated using atomic multiplet theory and the angular dependence is derived from crystal field symmetry. Applying our results to $\mathrm{Co} / \mathrm{NiO}(001)$ interfaces, we find perpendicular coupling between $\mathrm{Ni}$ and Co moments.
\end{abstract}

PACS numbers: $75.70 . \mathrm{Cn}, 78.70 . \mathrm{Dm}, 75.50 . \mathrm{Ee}, 75.70 . \mathrm{Ak}$ 
The detailed knowledge of the spin configuration in engineered magnetic nanostructures comprised of multiple layers with very different magnetic characteristics is essential to tailor their properties for device applications in information storage technology. For example, magnetic tunnel junctions used in magnetic random access memory [1] consist of two ferromagnetic layers with significantly different reversal fields separated by a thin insulating layer [2]. Exchange biased systems in which unidirectional anisotropy is induced in a ferromagnetic layer by an adjacent antiferromagnetic layer have found widespread use in read heads of hard drives [3]. Soft x-ray magnetic dichroism spectroscopies play an ever increasing role in improving our understanding of complex heteromagnetic nanostructures since these techniques provide elemental and chemical site specific magnetic information $[4,5]$ with high sensitivity [6] and tuneable probing depth [7]. X-ray spectromicroscopy techniques such as photoemission electron microscopy (PEEM) [8], scanning transmission x-ray microscopy (STXM) [9] or full field x-ray microscopy [10] add spatial resolution down to a few $\mathrm{nm}$ [11]. It is obvious that using spectroscopic information for magnetometry and magnetic microscopy, i.e., to determine the alignment of magnetic moments relative to the crystal axes and to image domains, requires the detailed knowledge and theoretical understanding of spectral shape and magnitude of dichroism signals as well as their dependence on the relative orientation of polarization, external field, and crystallographic axes.

In this Letter we show that the angular dependence of x-ray magnetic linear dichroism (XMLD) at the $\mathrm{Ni}^{2+} L_{2,3}$ edges in a lattice with cubic symmetry can only be described appropriately as linear combination of two fundamental spectra with distinct spectral features - not just one as previously proposed [12]. The fundamental spectra can be well reproduced using atomic multiplet theory while their angular dependence can be derived considering the symmetry of the crystal field. We present a general rule for the relative intensity of the two peaks in the $\mathrm{Ni} L_{2}$ edge frequently used to determine the antiferromagnetic axes in $\mathrm{NiO}$ systems [13-16]. Applying our results to exchange coupled $\mathrm{Co} / \mathrm{NiO}(001)$ interfaces we conclude that perpendicular coupling between $\mathrm{Ni}$ and Co moments is present as predicted for an almost perfectly compensated surface [17] and that an antiferromagnetic domain wall is formed in $\mathrm{NiO}$ upon magnetization reversal in the Co layer.

$40 \mathrm{~nm}$ thick $\mathrm{NiFe}_{2} \mathrm{O}_{4}$ films were prepared on polished $\mathrm{SrTiO}_{3}(011)$ and (001) substrates held at $725 \mathrm{~K}$ using pulsed laser deposition resulting in single crystalline films of proper stoichiometry. Characterization of the films will be reported elsewhere [18]. Moreover a 2.3 
nm thick Co film was deposited onto a freshly cleaved $\mathrm{NiO}(001)$ single crystal surface [13] and subsequently capped with $1 \mathrm{~nm} \mathrm{Pd}$. In both systems, $\mathrm{Ni}^{2+}$ ions are surrounded by $\mathrm{O}^{2-}$ ions in octahedral symmetry. The $\mathrm{x}$ ray absorption (XA) spectra, which are sensitive to the local environment, are very similar for both systems and can be described theoretically with atomic multiplet theory using similar parameter values. The angular dependence is determined by the crystal symmetry, which in both cases can be considered as cubic. The $\mathrm{Ni}^{2+}$ moments are coupled ferromagnetically in $\mathrm{NiFe}_{2} \mathrm{O}_{4}$ and can be aligned in any direction by external magnetic fields while antiferromagnetic coupling is present in $\mathrm{NiO}$. At $T=298 \mathrm{~K}$ all three samples can be saturated magnetically along any in plane direction by external magnetic fields of $0.5 \mathrm{~T}$. XA spectra were measured using the eight-pole resistive electromagnet [19] on beamline 4.0.2 at the Advanced Light Source (ALS) [20] providing (99 \pm 1$) \%$ linearly polarized $\mathrm{x}$ rays. All spectra were obtained in normal incidence at $T=298 \mathrm{~K}$ in electron yield mode, in the presence of external fields of $0.55 \mathrm{~T}$. The angular dependence of the XMLD signal across the $\mathrm{Ni} L_{2,3}$ edges was determined by rotating the orientation of $\mathrm{x}$ ray polarization $\mathbf{E}$ and external magnetic field $\mathbf{H}$ relative to the crystalline axes, c.f., inset to Fig. 1(a). E makes an angle $\varepsilon$ relative to the [100] crystal axis. The XMLD is the difference between XA spectra with $\mathbf{H}$ at angles $\mu$ and $\mu+90^{\circ}$ to the [100] direction, i.e., $I_{\mathrm{xmld}}(\varepsilon, \mu)=I_{\mathrm{xa}}(\varepsilon, \mu)-I_{\mathrm{xa}}\left(\varepsilon, \mu+90^{\circ}\right)$.

Figure 1(a) shows the Ni XA spectrum. In Fig. 1(b) the XMLD angular dependence of $\mathrm{NiFe}_{2} \mathrm{O}_{4} / \mathrm{SrTiO}_{3}(011)$ is displayed for $\varepsilon=\mu$ and $0^{\circ} \leq \mu \leq 90^{\circ}$, i.e., we measured the XMLD with $\mathbf{H}$ and hence the Ni moments parallel and perpendicular to $\mathbf{E}$ and varied the orientation of $\mathbf{E}$ relative to the crystal lattice. A strong anisotropy of the XMLD signal is observed. In particular, the $\mathrm{Ni} L_{2}$ XMLD signal reverses sign between $\varepsilon=0^{\circ}$ and $45^{\circ}$ and disappears almost completely for $\varepsilon=90^{\circ}$. This demonstrates that the spectral shape of the XMLD signal depends strongly on the orientations of $\mathbf{E}$ and $\mathbf{H}$ relative to the crystalline axes. It is essential to take this into account for a correct interpretation of the XMLD for magnetometry and microscopy applications.

Expressions for the angular dependence of the XMLD can be obtained from symmetry considerations [21] and it was shown that terms of higher order than $\cos 4 \phi$ can be ignored. 
For the geometry with $\mu=\varepsilon$ we have for the (001) and (011) planes in a cubic lattice

$$
\begin{aligned}
& I_{\text {xmld }}^{(001)}(\varepsilon)=\frac{1}{2}\left[I_{0}+I_{45}+\left(I_{0}-I_{45}\right) \cos 4 \varepsilon\right], \\
& I_{\text {xmld }}^{(011)}(\varepsilon)=I_{0}-\frac{1}{8}(5-2 \cos 2 \varepsilon-3 \cos 4 \varepsilon)\left(I_{0}-I_{45}\right)
\end{aligned}
$$

where $I_{0}$ and $I_{45}$ are defined as the spectra $I_{\text {xmld }}\left(\varepsilon=\mu=0^{\circ}\right)$ and $I_{\text {xmld }}\left(\varepsilon=\mu=45^{\circ}\right)$, respectively. Both these "fundamental spectra" are needed for a correct description of the angular dependence in cubic crystal field [21]. Figure 1(b) shows the modeled angular dependence using Eq. (2) and the experimental data for $I_{0}$ and $I_{45}$ in comparison with measured XMLD data. The agreement is excellent.

Additional confirmation was obtained from atomic multiplet calculations [22]. We fitted the experimental spectra using the calculated dipole transitions $\mathrm{Ni} 3 d^{8} \rightarrow 2 p^{5} 3 d^{9}$ in octahedral crystal field of $10 D q=1.4 \mathrm{eV}$ at specific directions of $\mathbf{E}$ and $\mathbf{H}$. The Hartree-Fock values of the atomic Slater integrals (listed in Ref. 22) were scaled to $75 \%$ to account for interatomic screening and the $2 p$ spin-orbit interaction scaled to $98 \%$. The obtained line spectra were convoluted with a Lorentzian of $\Gamma=0.1(0.4) \mathrm{eV}$ for the $L_{3}\left(L_{2}\right)$ edge to account for the intrinsic lifetime broadening and a Gaussian of $\sigma=0.15 \mathrm{eV}$ for the instrumental broadening. Figure 2 shows the calculated $I_{0}$ and $I_{45}$ in comparison with experimental data obtained from $\mathrm{NiFe}_{2} \mathrm{O}_{4} / \mathrm{SrTiO}_{3}(011)$ and (001). The agreement is remarkable. All experimentally observed features are reproduced by the calculation and only the intensity of the XMLD feature at $855.5 \mathrm{eV}$ appears to be overestimated by the calculation.

The XA spectra are determined by electric-dipole selection rules restricting the set of final states that can be reached from the ground state. This gives different transition probabilities from the exchange-split core levels to the $t_{2}$ and $e$ crystal-field-split empty $d$ states. The calculations show that the angular dependence of the XMLD signal disappears when the crystal field splitting goes to zero. Therefore, the anisotropic XMLD is a property of the cubic wavefunctions for the $d$ states with respect to the spin quantization axis and not of the anisotropic spin-orbit interaction [21].

The angular dependence of the $\mathrm{Ni} L_{2} \mathrm{XA}$ spectra in $\mathrm{NiO}(001)$ was characterized in detail by Alders et al. [12] and their result has been used extensively to study the $\mathrm{NiO}$ magnetic order in thin films and near interfaces [13-16]. The anisotropy of the XMLD signal was not observed due to the inability to change the orientation of Ni moments relative to the crystal lattice. $\mathrm{NiO} \mathrm{XA}$ spectra were interpreted assuming that the intensity of the second peak in 
the $\mathrm{Ni} L_{2}$ doublet structure is at a maximum when $\mathbf{E}$ is parallel to the antiferromagnetic axis $\mathbf{H}$ [12]. However, it follows from the results in Fig. 1 that, while this is indeed correct for $\varepsilon=0^{\circ}$, for $\varepsilon=45^{\circ}$ the second peak in the $\mathrm{Ni} L_{2}$ edge is also maximum when $\mathbf{E} \perp \mathbf{H}$. An equivalent statement is that $I_{45} \approx-I_{0}$ for the Ni $L_{2}$, c.f., Fig. 2(b). One should realize that this is a peculiarity of the $\mathrm{Ni}^{2+} L_{2}$ edge. It is not generally true for other transition metal $L_{2,3}$ edges $[21,23]$. The condition $I_{45}=-I_{0}$ at the $\mathrm{Ni}^{2+} L_{2}$ edge simplifies the angular dependence of the XA. In the plane at an angle $\theta$ to the (001) plane we have

$$
\begin{aligned}
I_{\mathrm{xa}}^{\theta}(\varepsilon, \mu)= & \frac{1}{2}\left[I_{\|}+I_{\perp}+\left(I_{\|}-I_{\perp}\right) \cos (2 \varepsilon+2 \mu)\right] \\
& -\left(I_{\|}-I_{\perp}\right) \sin ^{2} \varepsilon \sin ^{2} \mu \sin ^{2} 2 \theta
\end{aligned}
$$

where we define $I_{\|}=I_{\text {xa }}(\mathbf{E}\|\mathbf{H}\|[100])$ and $I_{\perp}=I_{\text {xa }}(\mathbf{E}\|[100], \mathbf{H}\|[010])$.

Maxima and minima for the second peak in the $L_{2}$ are reached when $I_{\text {xa }}=I_{\|}$and $I_{\perp}$, respectively. The maxima are confined to the $\{001\}$ planes, since the squared goniometric function in the last term of Eq. (3) is always positive, except for the (001) plane $\left(\theta=0^{\circ}\right)$ where it is zero.

For $\theta=0^{\circ}$, Eq. (3) gives the solution that $I_{\mathrm{xa}}=I_{\|}$if $\varepsilon=-\mu$ and that $I_{\mathrm{xa}}=I_{\perp}$ if $\varepsilon=-\mu+90^{\circ}$. Thus we can formulate a general rule stating that the maximum value is obtained when $\mathbf{H}$ and $\mathbf{E}$ are in an $\{001\}$ plane and have equal angles of opposite sign with the $\langle 100\rangle$ axis.

Instead of the XA, we can also use the XMLD. The difference spectrum has the advantage that the isotropic contribution, $I_{\|}+I_{\perp}$, in Eq. (3) cancels out. Using $I_{45}=-I_{0}$ together with the definition of the fundamental spectrum $I_{0}=I_{\|}-I_{\perp}$, the XMLD, obtained by rotating $\mathbf{H}$ over $90^{\circ}$ in the plane making an angle $\theta$ with (001), is

$$
I_{\text {xmld }}^{\theta}(\varepsilon, \mu)=I_{0}\left[\cos (2 \varepsilon+2 \mu)+\cos 2 \mu \sin ^{2} \varepsilon \sin ^{2} 2 \theta\right] .
$$

Thus for $\mathbf{H} \|[100], I_{\text {xmld }}^{\theta}=I_{0}\left(\cos 2 \varepsilon+\sin ^{2} \varepsilon \sin ^{2} 2 \theta\right)$ vanishes in the (001) and (011) planes at $\varepsilon=45^{\circ}$ and $90^{\circ}$, respectively, which demonstrates the strong anisotropy.

The observation of anisotropic Ni XMLD requires revisiting previous experiments interpreted without considering the anisotropy. For example, Ohldag et al. [13] observe a reorientation of $\mathrm{Ni}$ moments to inplane for the surface near region of $\mathrm{NiO}(001)$ upon $\mathrm{Co}$ deposition. For domains with Co moments aligned parallel to the [110] direction, the second peak in the $\mathrm{Ni} L_{2}$ shows a maximum for $\mathbf{E} \|[110]$ indicating that the Ni moments are 
aligned perpendicular to the [110] direction. Consequently, the coupling between $\mathrm{Ni}$ and Co moments is perpendicular — not parallel.

To investigate the interfacial coupling in $\mathrm{Co} / \mathrm{NiO}(001)$ further we employed exchange coupling between $\mathrm{Co}$ and $\mathrm{Ni}$ moments at the $\mathrm{Co} / \mathrm{NiO}$ interface to create an antiferromagnetic exchange spring near the $\mathrm{NiO}$ surface by aligning the Co moments parallel to an external magnetic field [16]. We measured the $\mathrm{Ni}$ XMLD in $\mathrm{NiO}$ through alignment of the $\mathrm{Co}$ moments with an external magnetic field of $0.55 \mathrm{~T}$ at angles $\mu$ and $\mu+90^{\circ}$ to the [100] direction, while $\mathbf{E}$ is at an angle $\varepsilon$ to [100], c.f., inset to Fig. 3. Figure 3(b) shows the angular dependence obtained with $\varepsilon=\mu$. Due to the perpendicular coupling between Co and $\mathrm{Ni}$ moments, the $\mathrm{Ni}$ moments are always aligned perpendicular to the external field. Hence the observed XMLD signal corresponds to $I_{\mathrm{xmld}}^{\mathrm{NiO}}(\varepsilon, \mu)=I_{\text {xmld }}^{\mathrm{NiFe}_{2} \mathrm{O}_{4}}\left(\varepsilon, \mu+90^{\circ}\right)$. We can take this into account by defining the fundamental spectra as $I_{0}^{\mathrm{NiO}}=-I_{0}^{\mathrm{NiFe}_{2} \mathrm{O}_{4}}$ and $I_{45}^{\mathrm{NiO}}=$ $-I_{45}^{\mathrm{NiFe}_{2} \mathrm{O}_{4}}$. The fundamental spectra obtained for $\mathrm{Co} / \mathrm{NiO}(001)$ are compared in Fig. 2(b) with those observed for $\mathrm{NiFe}_{2} \mathrm{O}_{4} / \mathrm{SrTiO}_{3}(011)$ and (001) and the atomic multiplet results. Within the experimental accuracy the measured spectra are identical. We can model the XMLD angular dependence using Eq. (1) with the experimental $I_{0}$ and $I_{45}$. Figure 3(b) shows that, as in the case of $\mathrm{NiFe}_{2} \mathrm{O}_{4} / \mathrm{SrTiO}_{3}(011)$, the agreement between experiment and model is very good.

Figure 3(c) shows the anisotropy of the Ni XMLD measured with $\mathbf{E}$ fixed at $\varepsilon=0^{\circ}$ and varying the orientation of $\mathbf{H}$, in which case Eq. (4) gives $I_{\mathrm{xmld}}\left(\varepsilon=0^{\circ}\right)=-I_{0} \cos 2 \mu$. Thus the spectral shape of the XMLD is independent of $\mu$ and changes only in magnitude. There is an excellent agreement of experimental data and the modeled angular dependence. Figure 3(d) displays experimental data and modeled XMLD spectra obtained for $\varepsilon=45^{\circ}$ and $0^{\circ} \leq \mu \leq 90^{\circ}$. The angular dependence is very well described by $I_{\mathrm{xmld}}^{(001)}\left(\varepsilon=45^{\circ}\right)=-I_{45} \sin 2 \mu$. In summary, we studied the anisotropy of XMLD at the $\mathrm{Ni} L_{2,3}$ edges in $\mathrm{NiFe}_{2} \mathrm{O}_{4} / \mathrm{SrTiO}_{3}(011)$ and (001), and $\mathrm{Co} / \mathrm{NiO}(001)$. The XMLD angular dependence is a linear combination of two fundamental spectra with distinct spectral features. The fundamental spectra share the same spectral shapes for the ferromagnet and antiferromagnet (but not the same magnitude). These spectra are well reproduced using atomic multiplet theory while their angular dependence can be derived considering the symmetry of the crystal field. So even if no experimental data is available theory can help taking the XMLD anisotropy into account. We present a general rule for the relative intensities of the double peak feature 
at the $\mathrm{Ni} L_{2}$ edge often used to determine the antiferromagnetic axes in $\mathrm{NiO}$ systems. We apply our method by revisiting the exchange coupled $\mathrm{Co} / \mathrm{NiO}(001)$ interface where we observe perpendicular coupling between $\mathrm{Ni}$ and Co moments as predicted by theory [17] and the formation of an antiferromagnetic domain wall upon magnetization reversal in the Co layer.

The ALS is supported by the Department of Energy. We thank Andreas Scholl for supplying the $\mathrm{Co} / \mathrm{NiO}$ sample.

* Electronic address: earenholz@lbl.gov

$\dagger$ Electronic address: g.vanderlaan@dl.ac.uk

¥ Also at School of Applied Physics, Cornell University, Ithaca, NY 14853

[1] S. S. P. Parkin et al., Proc. IEEE 91, 661 (2003).

[2] S. S. P. Parkin et al., J. Appl. Phys. 85, 5828 (1999).

[3] A. E. Berkowitz and K. Takano, J. Magn. Magn. Mater. 200, 552 (1999).

[4] B. T. Thole, G. van der Laan, and G. A. Sawatzky, Phys. Rev. Lett. 55, 2086 (1985).

[5] G. van der Laan et al., Phys. Rev. B 34, 6529 (1986).

[6] M. G. Samant et al., Phys. Rev. Lett. 72, 1112 (1994).

[7] Y. U. Idzerda et al., Nucl. Instrum. Meth. Phys. Res. A 347, 134 (1994).

[8] B. P. Tonner et al. et al., Nucl. Instrum. Meth. Phys. Res. A 347, 142 (1994).

[9] T. Warwick et al., Rev. Sci. Instrum. 69, 2964 (1998).

[10] P. Fischer et al., Rev. Sci. Instrum. 72, 2322 (2001).

[11] W. Chao et al., Nature 435, 1210 (2005).

[12] D. Alders et al., Phys. Rev. B 57, 11623 (1998).

[13] H. Ohldag et al., Phys. Rev. Lett. 86, 2878 (2001).

[14] J. Stohr et al., Phys. Rev. Lett. 83, 1862 (1999).

[15] H. Ohldag et al., Phys. Rev. Lett. 87, 247201 (2001).

[16] A. Scholl et al., Phys. Rev. Lett. 92, 247201 (2004).

[17] T. C. Schulthess and W. H. Butler, Phys. Rev. Lett. 81, 4516 (1998).

[18] R. V. Chopdekar, E. Arenholz, and Y. Suzuki, to be published (2007).

[19] E. Arenholz and S. O. Prestemon, Rev. Sci. Instrum. 76, 083908 (2005). 
[20] A. T. Young et al., J. Synchrotron Rad. 9, 270 (2002).

[21] E. Arenholz et al., Phys. Rev. B 74, 094407 (2006).

[22] G. van der Laan and B. T. Thole, Phys. Rev. B 43, 13401 (1991).

[23] A. A. Freeman et al., Phys. Rev. B 73, 233303 (2006). 


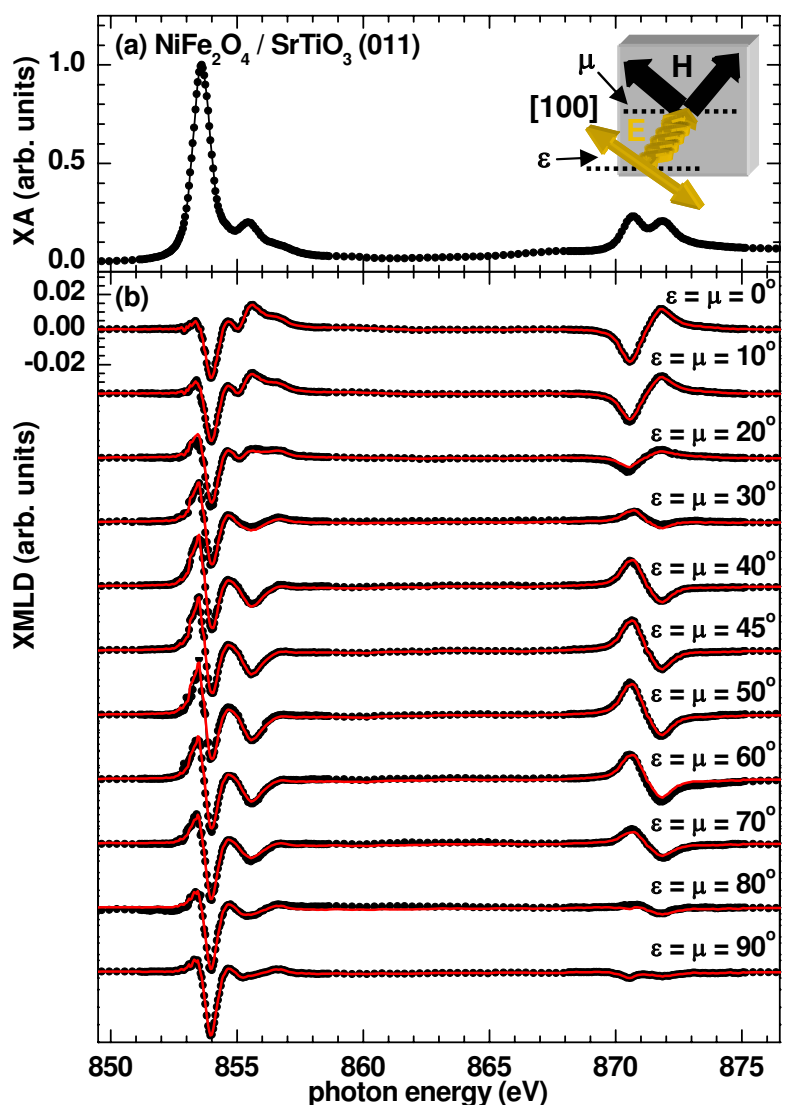

FIG. 1: (Color online) Angular dependence of the $\mathrm{Ni} L_{2,3} \mathrm{XMLD}$ in $\mathrm{NiFe}_{2} \mathrm{O}_{4} / \mathrm{SrTiO}_{3}(011)$. The inset depicts the experimental geometry. The XMLD spectra are obtained as difference between two XA spectra measured in applied external fields $\mathbf{H}$ (black arrows) at angles $\mu$ and $\mu+90^{\circ}$ relative to the [100] axis (dashed line). The $\mathrm{x}$ rays impinge on the sample in normal incidence with linear polarization $\mathbf{E}$ (yellow arrow) at an angle $\varepsilon$ to the [100] axis. (a) XA spectrum averaged over all angles $\varepsilon$. (b) XMLD spectra for $\varepsilon=\mu$ with $0^{\circ} \leq \mu \leq 90^{\circ}$. Symbols indicate the experimental data and (red) lines give the results of the modeled angular dependence. 


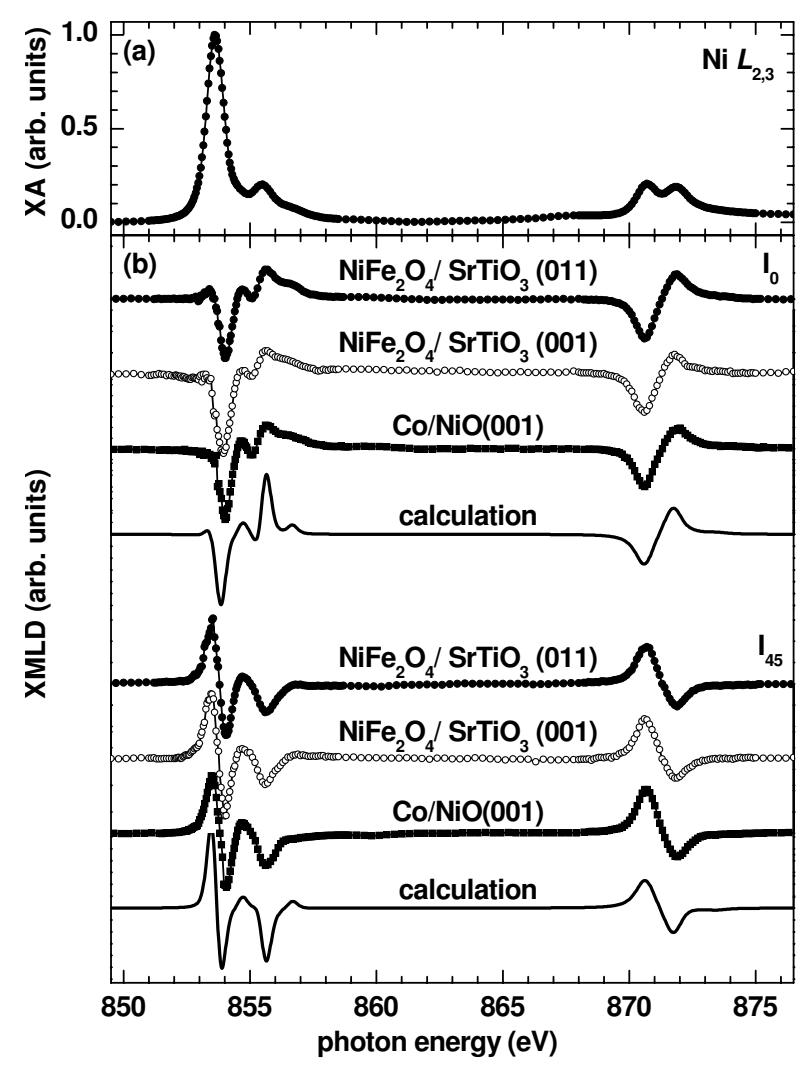

FIG. 2: Comparison of the experimentally obtained fundamental XMLD spectra together with results from atomic multiplet calculations. (a) Measured $\mathrm{Ni} L_{2,3} \mathrm{XA}$ spectrum for $\mathrm{NiFe}_{2} \mathrm{O}_{4} / \mathrm{SrTiO}_{3}$. (b) Fundamental XMLD spectra $I_{0}$ i.e., $\varepsilon=\mu=0^{\circ}$ (upper four spectra) and $I_{45}$ i.e., $\varepsilon=\mu=45^{\circ}$ (lower four spectra). Experimental data from $\mathrm{NiFe}_{2} \mathrm{O}_{4} / \mathrm{SrTiO}_{3}(011)$ (solid circles), $\mathrm{NiFe}_{2} \mathrm{O}_{4} / \mathrm{SrTiO}_{3}(001$ ) (open circles) and $\mathrm{Co} / \mathrm{NiO}(001)$ (solid squares). The multiplet calculations are shown by solid lines. 


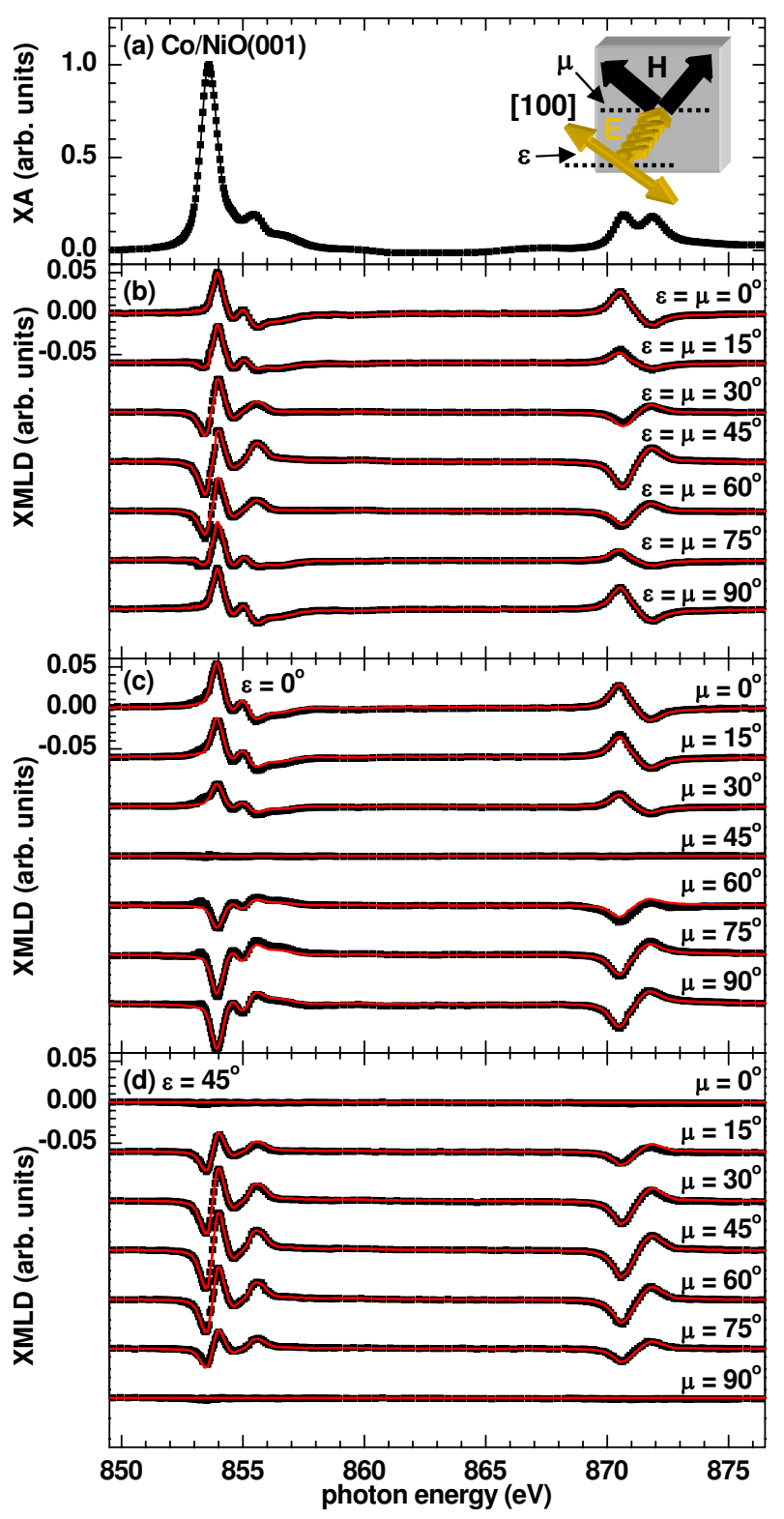

FIG. 3: (Color online) Angular dependence of Ni $L_{2,3}$ XMLD for Co/NiO(001). Solid symbols indicate experimental data while (red) lines show results of the modeled angular dependence. (a) Ni $L_{2,3}$ XA spectrum. The inset shows the experimental geometry as described in Fig. 1(a). XMLD spectra obtained for $0^{\circ} \leq \mu \leq 90^{\circ}$ with (b) $\varepsilon=\mu$, (c) $\varepsilon=0^{\circ}$, and (d) $\varepsilon=45^{\circ}$. 